LUNG CANCER

\title{
Comparison of shuttle walk with measured peak oxygen consumption in patients with operable lung cancer
}

\author{
T Win, A Jackson, A M Groves, L D Sharples, S C Charman, C M Laroche
}

Thorax 2006;61:57-60. doi: 10.1136/thx.2005.043547

See end of article for authors' affiliations

Correspondence to:

Dr T Win, Lister Hospital,

Stevenage, Hertfordshire

SGI $4 A B$, UK;

drthidawin@hotmail.com

Received 8 March 2005

Accepted 10 October 2005

Published Online First

21 October 2005
Background: The relationship between the shuttle walk test and peak oxygen consumption in patients with lung cancer has not previously been reported. A study was undertaken to examine this relationship in patients referred for lung cancer surgery to test the hypothesis that the shuttle walk test would be useful in this clinical setting.

Methods: 125 consecutive patients with potentially operable lung cancer were prospectively recruited. Each performed same day shuttle walking and treadmill walking tests.

Results: Shuttle walk distances ranged from $104 \mathrm{~m}$ to $1020 \mathrm{~m}$ and peak oxygen consumption ranged from 9 to $35 \mathrm{ml} / \mathrm{kg} / \mathrm{min}$. The shuttle walk distance significantly correlated with peak oxygen consumption $(r=0.67, \mathrm{p}<0.001)$. All 55 patients who achieved more than $400 \mathrm{~m}$ on the shuttle test had a peak oxygen consumption of at least $15 \mathrm{ml} / \mathrm{kg} / \mathrm{min}$. Seventy of 125 patients failed to achieve $400 \mathrm{~m}$ on the shuttle walk test; in 22 of these the peak oxygen consumption was less than $15 \mathrm{ml} / \mathrm{kg} / \mathrm{min}$. Nine of 17 patients who achieved less than $250 \mathrm{~m}$ had a peak oxygen consumption of more than $15 \mathrm{ml} / \mathrm{kg} / \mathrm{min}$.

Conclusion: The shuttle walk is a useful exercise test to assess potentially operable lung cancer patients with borderline lung function. However, it tends to underestimate exercise capacity at the lower range compared with peak oxygen consumption. Our data suggest that patients achieving $400 \mathrm{~m}$ on the shuttle walk test do not require formal measurement of oxygen consumption. In patients failing to achieve this distance we recommend assessment of peak oxygen consumption, particularly in those unable to walk $250 \mathrm{~m}$, because a considerable proportion would still qualify for surgery as they had an acceptable peak oxygen consumption.
S urgery remains the treatment of choice for resectable lung carcinoma with respect to curability. However, some surgical patients have a poor postoperative outcome, usually due to the presence of co-existing disease, especially chronic obstructive pulmonary disease (COPD). To assist the prediction of surgical outcome, a range of cardiopulmonary exercise tests have been used. These include maximal exercise tests, full lung function, and walk tests.

A number of studies have suggested that cardiopulmonary exercise testing is one of the most valuable parameters for the evaluation of risk assessment in lung cancer surgery. ${ }^{1-5}$ It provides the best index of functional capacity and global oxygen transport/consumption $\left(\mathrm{VO}_{2}\right)$, as well as estimating both cardiac and pulmonary reserve not available from other modalities. However, cardiopulmonary exercise testing is often poorly available in Europe.

The 10 metre shuttle walk test is a standardised, externally paced, incremental and progressive field test which is readily available in most hospitals. It is a reproducible measure of functional capacity in patients with chronic airflow limitation. ${ }^{6}$ In patients with COPD the shuttle walk test has been shown to be closely correlated with $\mathrm{VO}_{2}$ max. ${ }^{7}$ However, there are no data comparing the relationship between shuttle walk distance and $\mathrm{VO}_{2}$ peak in patients with lung cancer. These data are necessary as many patients with bronchogenic carcinoma do not have COPD and may therefore have different respiratory physiology.

In the selection of patients for lung cancer surgery, both the British Thoracic Society (BTS $)^{8}$ and American College of Chest Physicians (ACCP) ${ }^{9}$ suggest that all patients with borderline lung function and with predicted postoperative forced expiratory volume in 1 second $\left(\mathrm{FEV}_{1}\right)$ or carbon monoxide transfer factor (TLCO) of less than $40 \%$ should undergo exercise testing before surgery. However, the scientific rationale for this strategy is rather vague. In those patients who subsequently undergo shuttle walk testing and fail to complete 250 metres, it is recommended that surgery should not be attempted. ${ }^{8}$ This is because it is believed that failure to achieve this distance would predict a $\mathrm{VO}_{2}$ max of less than $10 \mathrm{ml} / \mathrm{kg} / \mathrm{min}$ and thus a high risk of poor postoperative outcome. The basis of this prediction between shuttle walk distance and oxygen consumption originates from work using the regression equation developed by Singh et al. ${ }^{7}$ However, Singh's study population was small and all the patients had COPD so it does not necessarily reflect the lung cancer patient population. Indeed, only limited data are available on the ability of the shuttle walk test to predict oxygen consumption. ${ }^{10}$

A prospective study was therefore performed to examine the relationship between the shuttle walk test and oxygen consumption specifically in preoperative lung cancer patients with both normal and borderline lung function.

\section{METHODS}

\section{Patients}

125 consecutive patients with potentially resectable lung cancer (81 men) of mean age 69 years (range 42-85) were prospectively recruited. Forty one (33\%) had borderline lung function $\left(\mathrm{FEV}_{1}<1.5 \mathrm{l}\right.$ for lobectomy and $\mathrm{FEV}_{1}<2.0 \mathrm{l}$ for pneumonectomy). Exclusion criteria included unstable

Abbreviations: $\mathrm{FEV}_{1}$, forced expiratory volume in 1 second; $\mathrm{KCO}$, carbon monoxide transfer coefficient; TLCO, carbon monoxide transfer factor, $\mathrm{VO}_{2}$ peak, peak oxygen consumption 
angina, recent myocardial infarction (within 6 weeks), and disorders that might influence exercise performance physically such as severe back pain. The local ethics committee approved the study and all patients gave informed consent.

The shuttle walking and treadmill walking tests were performed on the same day, at least 4 hours apart. The patients were fully familiarised with both tests before the start. All patients also underwent full pulmonary function tests according to the guidelines of the Association of Respiratory Technology and Physiology. ${ }^{11}$ Follow up data were analysed 12 months after surgery.

\section{Shuttle walking test}

The shuttle walking test was performed using methods established by Singh et al. ${ }^{6}$ Patients walked between two cones 10 metres apart at an incrementally increasing pace. Each increment was signalled by a fully calibrated audiocassette. To assist, the operator accompanied the patient throughout the test. The end point was reached when the patient could no longer maintain the required speed or became too breathless to proceed further. Using a pulse oximeter (Minolta Pulsox 3i), oxygen saturation and heart rate were recorded every 30 seconds throughout the test.

The Borg breathlessness score ${ }^{12}$ was also recorded at the beginning and end of the test. A note of the recovery time and the reason for terminating the shuttle walk were also recorded.

\section{Cardiopulmonary exercise test $\left(\mathrm{VO}_{2}\right.$ test)}

The cardiopulmonary exercise test was performed using the Oxycon-Pro Exercise System Viasys Healthcare with the Standardized Exponential Exercise Protocol (STEEP) as described by Northridge et al, ${ }^{13}$ with the exception of an additional 1 minute warm up period. The STEEP protocol was devised to allow a single protocol to be selected for subjects with a wide range of exercise capacities which could be applied to the treadmill. The protocol commenced at a low workload and was increased exponentially by $15 \%$ every minute. The treadmill protocol involved changes in either gradient or speed, but never both simultaneously.

The test lasted a maximum of 20 minutes, of which the patient exercised for 16 minutes and 4 minutes were taken up with baseline measurements and recovery. Patients were required to exercise for as long as possible until they were sign and/or symptom restricted. The ECG was monitored throughout the test, and oxygen and carbon dioxide were measured from the expired air. The Borg breathlessness score ${ }^{12}$ and the reason for terminating the test were also recorded.

Three $\mathrm{VO}_{2}$ values measured in the final 30 seconds of the exercise phase were averaged to obtain the $\mathrm{Vo}_{2}$ peak. We defined the oxygen consumption as $\mathrm{VO}_{2}$ peak rather than $\mathrm{VO}_{2} \max$ since $\mathrm{VO}_{2} \max$ is usually obtained from the maximum oxygen consumption during the plateau stage of exercise in healthy subjects. However, in many patients it is often difficult to maintain this plateau stage of exercise, so in our patients $\mathrm{VO}_{2}$ peak is considered more useful and not significantly different from $\mathrm{VO}_{2}$ max. ${ }^{14}$

\section{Statistical analysis}

The baseline characteristics are presented as mean (SD) values. The Student's $t$ test was used for subgroup comparisons of these measurements. Pearson's correlation coefficients were used to assess the associations between measurements of respiratory physiology and exercise capacity. In those patients who underwent surgery, survival time was measured from the date of the procedure to the date of death and was censored at 15 August 2005 for surviving patients. Kaplan-Meier survival rates were estimated and compared using a log rank test. Receiver operating
Table 1 Baseline characteristics and lung function values

\begin{tabular}{|c|c|c|}
\hline & Mean (SD) & Range \\
\hline Age & $68.8(7.7)$ & $42-85$ \\
\hline $\mathrm{FEV}_{1}(\mathrm{l})$ & $1.97(0.63)$ & $0.5-4.3$ \\
\hline $\mathrm{FEV}_{1}$ (\% predicted) & $75.7 \%(21.1)$ & $40-119$ \\
\hline Kco (\% predicted) & $84.4 \%(21.5)$ & $37-111$ \\
\hline TLCO (\% predicted) & $70.9 \%(18.8)$ & $35-106$ \\
\hline Shuttle distance (m) & $403(143.3)$ & $60-1020$ \\
\hline $\mathrm{VO}_{2}$ peak $(\mathrm{ml} / \mathrm{kg} / \mathrm{min})$ & $18.3(4.5)$ & $9-35$ \\
\hline $\mathrm{VO}_{2}$ peak (\% predicted) & $84.3(30.8)$ & $44.3-189$ \\
\hline
\end{tabular}

$\mathrm{FEV}_{1}$, forced expiratory volume in 1 second; $\mathrm{KCO}$, carbon monoxide transfer coefficient; TLCO, carbon monoxide transfer factor; $\mathrm{VO}_{2}$ peak, peak oxygen consumption.

characteristic (ROC) curves were used to assess the value of the shuttle walk test on 12 month survival in patients who underwent surgery. The Wilcoxon test was used to assess whether the area under the ROC curve was significantly greater than 0.5 .

\section{RESULTS}

All patients completed the study. Baseline characteristics, $\mathrm{FEV}_{1}, \mathrm{FEV}_{1} \%$ predicted, TLCO \% predicted, Kco \% predicted, shuttle walk distance, $\mathrm{VO}_{2}$ peak, and $\mathrm{VO}_{2}$ peak $\%$ predicted are shown in table 1 . Shuttle walk distances ranged from $60 \mathrm{~m}$ to $1020 \mathrm{~m}$ (mean $400 \mathrm{~m}$ ). During the walk, mean desaturations of $0-14 \%$ were recorded. Changes in Borg score ranged from 0 to 6 (mean 2.3). $\mathrm{Vo}_{2}$ peak ranged from 9 to $35 \mathrm{ml} / \mathrm{kg} / \mathrm{min}$ (mean $18.3 \mathrm{ml} / \mathrm{kg} / \mathrm{min}$ ); $44.3-189 \%$ of normal predicted (mean 84\%).

Fifty five of the 125 patients achieved more than $400 \mathrm{~m}$ on the shuttle test, all of whom had a measured $\mathrm{VO}_{2}$ peak of $\geqslant 15 \mathrm{ml} / \mathrm{kg} / \mathrm{min}$. Seventy of the 125 patients failed to achieve $400 \mathrm{~m}$ on the shuttle walk test; in 22 of these patients (31.5\%) the $\mathrm{VO}_{2}$ peak was $<15 \mathrm{ml} / \mathrm{kg} / \mathrm{min}$. Seventeen patients achieved less than $250 \mathrm{~m}$ on the shuttle walk test, nine of whom had a $\mathrm{VO}_{2}$ peak of $>15 \mathrm{ml} / \mathrm{kg} / \mathrm{min}$ while 16 of the 17 had a $\mathrm{VO}_{2}$ peak of $>10 \mathrm{ml} / \mathrm{kg} / \mathrm{min}$.

The maximum heart rate ranged from 77 to 185 beats/min. No significant arrhythmias were recorded during the tests. All patients had a recovery time (time to return to pre-exercise oxygen saturation and Borg score) of less than 4 minutes. No adverse effects were noted. Two patients developed significant ST changes ( $\geqslant 1 \mathrm{~mm}$ ST elevation or depression) without symptoms; they were referred for coronary angiography which confirmed significant coronary artery disease. Table 2 shows the reasons for stopping exercise tests.

There was a significant correlation between shuttle walk distance and $\mathrm{VO}_{2}$ peak $(r=0.67, \mathrm{p}<0.001$; fig 1$)$, but the correlation between shuttle walk distance and $\mathrm{VO}_{2}$ peak \% predicted was weak $(r=0.30)$. There was a moderate correlation between actual $\mathrm{Vo}_{2}$ peak and $\mathrm{FEV}_{1}(r=0.49)$ and between shuttle walk distance and $\operatorname{FEV}_{1}(r=0.5)$. There was no significant correlation between oxygen saturation and Borg score $(r=0.10)$ or between oxygen saturation and shuttle walk distance $(r=0.063)$. There was moderate

Table 2 Reasons for stopping exercise tests

\begin{tabular}{lll}
\hline & $\begin{array}{l}\mathrm{Vo}_{2} \text { max } \\
\text { treadmill test }\end{array}$ & $\begin{array}{l}\text { Shuttle walk } \\
\text { test }\end{array}$ \\
\hline Leg pain/fatigue & $48 \%$ & $16 \%$ \\
Unable to keep up with speed & $10 \%$ & $70 \%$ \\
ECG changes & $4 \%$ & $\mathrm{NA}$ \\
Chest discomfort & $2 \%$ & $6 \%$ \\
Breathlessness & $36 \%$ & $8 \%$ \\
\hline
\end{tabular}




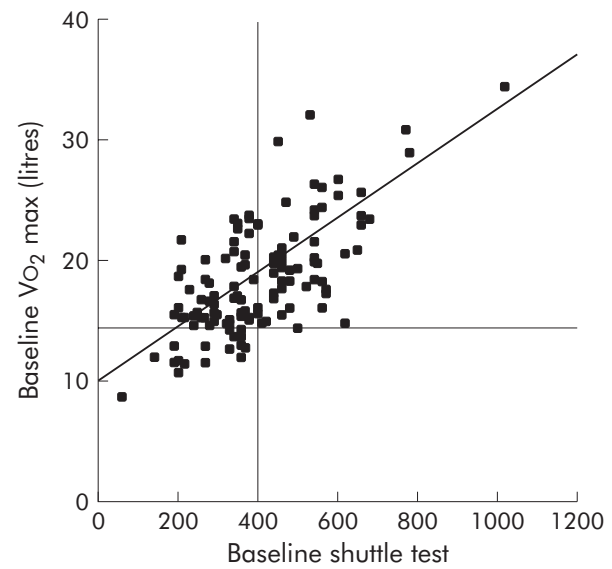

Figure 1 Correlation $(r=0.67)$ between shuttle walk distance $(\mathrm{m})$ and peak oxygen consumption ( $\mathrm{VO}_{2}$ peak, $\left.\mathrm{ml} / \mathrm{kg} / \mathrm{min}\right)$.

correlation between shuttle walk distance and change in Borg score $(r=0.33)$.

The relationship between shuttle walk distance and $\mathrm{VO}_{2}$ peak was the same in patients with normal lung function as in those with borderline pulmonary function. Forty three patients whose $\mathrm{FEV}_{1}$ was considered adequate for lung cancer surgery (according to the guidelines) could not achieve $400 \mathrm{~m}$ on the shuttle walk test. There was no statistically significant association between lung function tests $\left(\mathrm{FEV}_{1}\right.$ or $\left.\mathrm{FEV}_{1} / \mathrm{VC} \%\right)$ and either $\mathrm{VO}_{2}$ peak or shuttle walk distance (table 3 ).

The final decision to operate was taken in the multidisciplinary team meeting, depending on physiological data, co-morbidity, and cardiothoracic surgical opinion. The reason for non-operation was upstaging in $86 \%$ (for example, after positron emission tomography) and $14 \%$ were excluded for co-morbidity (for example, heart disease or poor respiratory reserve secondary to COPD).

Of the 100 patients with a $\mathrm{VO}_{2}$ peak of $\geqslant 15 \mathrm{ml} / \mathrm{kg} / \mathrm{min}, 85$ had surgery. At 1 year after surgery 69 of the $85(81 \%)$ had survived. Of the 25 patients with $\mathrm{VO}_{2}$ peak of $<15 \mathrm{ml} / \mathrm{kg} / \mathrm{min}$, 17 had surgery and at 1 year nine of them (53\%) had survived. The mean (SD) $\mathrm{VO}_{2}$ peak in those who survived 1 year after surgery was 19.5 (4.4) $\mathrm{ml} / \mathrm{kg} / \mathrm{min}$ compared with 16.9 (4.1) $\mathrm{ml} / \mathrm{kg} / \mathrm{min}$ in those who died $(\mathrm{p}=0.013)$. Figure 2 shows the ROC curve for shuttle walk test against 1 year survival in patients who underwent surgery. The area under the curve is $0.70(p=0.003)$, indicating that the shuttle walk test has significant predictive value. A threshold of $400 \mathrm{~m}$ in the shuttle walk test had a sensitivity of $77 \%$ and specificity of $54 \%$ for 1 year survival. One year after surgery, 42 (88\%) of 48 patients who achieved a shuttle walk distance of $400 \mathrm{~m}$ or more had survived compared with 36 of 54 (67\%) of those who did not achieve $400 \mathrm{~m}$ (fig 3). The mean (SD) shuttle walk distance in those who survived to l year was 428 (135) $\mathrm{m}$ compared with 335 (122) $\mathrm{m}$ in those who died $(\mathrm{p}=0.003)$.

\section{DISCUSSION}

We have shown that the relationship between $\mathrm{VO}_{2}$ peak and shuttle walk distance is significantly closer than the relationship between $\mathrm{Vo}_{2}$ peak and any other parameters of lung function. In addition, the relationship between shuttle walk distance and $\mathrm{VO}_{2}$ peak was the same in patients with normal lung function as in those with borderline pulmonary function. In fact, many patients with acceptable lung function according to the guidelines failed to achieve $400 \mathrm{~m}$ on the shuttle walk test, and this could imply that the use of static lung function tests in this setting might be limited. Shuttle walk distance and change in the Borg breathlessness

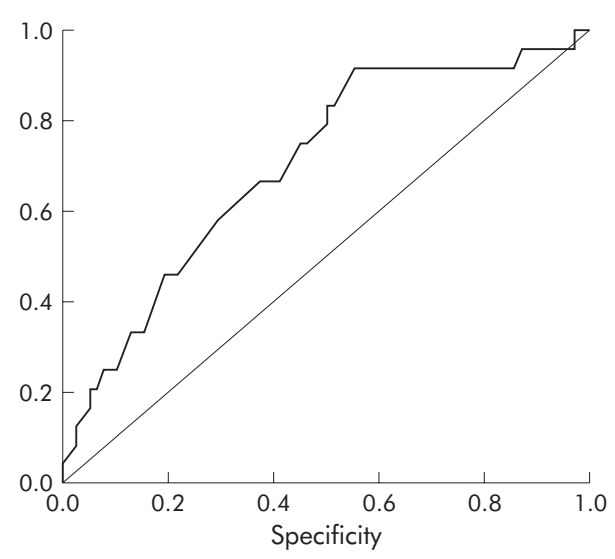

Figure 2 Receiver operating characteristic curve for shuttle walk test distance against 12 month survival in patients who underwent surgery. The area under the curve is $0.70(p=0.003)$.

score had a weak correlation. The reason for terminating the test was mainly speed limitation in the shuttle walk test, whereas symptoms such as leg pain were the main cause for cessation on the treadmill. There was a weak correlation between shuttle walk distance and $\mathrm{Vo}_{2}$ peak percentage predicted. As there is no established predicted shuttle walk distance for each individual, we are unable to correlate the percentage predicted of these two different tests.

Although we found there to be significant correlation between shuttle walk distance and $\mathrm{VO}_{2}$ peak $(r=0.67 ; 95 \%$ confidence interval 0.56 to $0.76 ; \mathrm{n}=125$ ), the correlation was weaker than that claimed by Singh et al $(r=0.88, \mathrm{n}=19){ }^{7}$ The reason for this difference is multifactorial. Singh's cohort was younger, predominantly male, and all suffered from COPD rather than lung cancer. Their patients also had lower $\mathrm{VO}_{2}$ peak and $\mathrm{FEV}_{1}$ values. In addition, our patient group was likely to have been better motivated by the incentive of curable surgery.

There is evidence that tests such as the modified shuttle walk test, ${ }^{15}$ Cooper walk run test, ${ }^{16}$ and the multi-stage shuttle run test ${ }^{17}$ correlate better with oxygen consumption than the shuttle walk test. However, these procedures require vigorous exercise and, as such, would be inappropriate to perform in our patients who are usually elderly and frequently suffer from chronic lung disease. Another walk test often used is the 6 minute walk test. However, interpretation of the distance

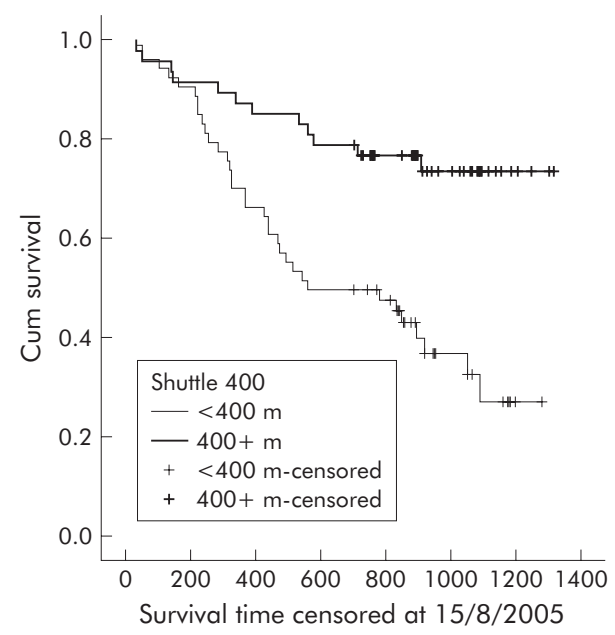

Figure 3 Kaplan-Meier survival curve showing the survival advantage of patients achieving $400 \mathrm{~m}$ in the shuttle walk test compared with those who walked less than $400 \mathrm{~m}$. 
Table 3 Correlation between shuttle walk distance, peak oxygen consumption ( $\mathrm{VO}_{2}$ peak) and lung function

\begin{tabular}{lllllll}
\hline & Shuttle & $\mathrm{Vo}_{2}$ max & $\mathrm{Vo}_{2} \max (\%)$ & $\mathrm{FEV}_{\mathbf{1}}$ & $\mathrm{Kco}(\%)$ & TLCo $(\%)$ \\
\hline Shuttle & 1 & 0.67 & 0.30 & 0.50 & 0.24 & 0.39 \\
Vo o $_{2}$ peak & 0.67 & 1 & 0.43 & 0.50 & 0.22 & 0.44 \\
\hline
\end{tabular}

FEV 1 , forced expiratory volume in 1 second; TLCO, carbon monoxide transfer factor; $\mathrm{KCO}$, carbon monoxide transfer coefficient; $\mathrm{VO}_{2}$ peak, peak oxygen consumption.

walked in 6 minutes is currently not well standardised. ${ }^{18}$ Although the stair climbing test has been used historically as a surrogate for cardiopulmonary exercise test, ${ }^{19}$ it is not performed in standardised manner. The shuttle walk test has been shown to be reproducible and a good predictor of the impact of breathlessness on functional capacity in patients with cancer. ${ }^{20}$ We therefore chose to perform the shuttle walk test as a reliable field test on our patients.

We used the STEEP protocol as it had numerous advantages for our patient cohort and has been validated against a wide range of patients with different exercise capacities. ${ }^{13}$ This was important as $33 \%$ of our patients had borderline lung function. The STEEP protocol has also been validated in patients with coronary artery disease $\mathrm{e}^{21}$ and a number of our patients had evidence of myocardial ischaemia. It also benefits from versatility as it can be used with either exercise bike or treadmill.

A correlation of 0.67 between the shuttle walk distance and $\mathrm{VO}_{2}$ peak has possible implications for both the BTS and ACCP guidelines for selecting patients for lung cancer surgery. At present it is recommended that patients who cannot walk $250 \mathrm{~m}$ on the shuttle walk test need not undergo $\mathrm{Vo}_{2}$ measurements as it is assumed that the $\mathrm{VO}_{2} \max$ would be less than $10 \mathrm{ml} / \mathrm{kg} / \mathrm{min}$ and that therefore they would be in a high risk group for surgery. However, these recommendations appear to have come from limited data on patients with COPD. ${ }^{7}$ Our findings suggest that, if the present guidelines are adhered to with respect to the $250 \mathrm{~m}$ cut off, then some patients would needlessly be excluded from surgery. In our study nine patients who failed to reach $250 \mathrm{~m}$ had a measured $\mathrm{VO}_{2}$ peak which exceeded $15 \mathrm{ml} / \mathrm{kg} / \mathrm{min}$ and thus would meet the criteria for surgery. In fact, with one exception, all patients who failed to reach $250 \mathrm{~m}$ had $\mathrm{VO}_{2}$ peak measurements of $>10 \mathrm{ml} / \mathrm{kg} / \mathrm{min}$.

Our experience showed that all patients who exceeded $400 \mathrm{~m}$ on the shuttle walk test had $\mathrm{VO}_{2}$ peak measurements of $>15 \mathrm{ml} / \mathrm{kg} / \mathrm{min}$. One can therefore argue that measurement of $\mathrm{VO}_{2}$ in these patients is unnecessary since a $\mathrm{VO}_{2}$ peak of $>15 \mathrm{ml} / \mathrm{kg} / \mathrm{min}$ has been shown to be quite safe for resections. Indeed, the survival data would seem to confirm the usefulness of the $400 \mathrm{~m}$ cut off. Although this finding may not benefit all patients, a sizeable cohort would be saved an invasive test and these patients could be investigated outside tertiary care centres. Patients undergoing complete pneumonectomy may need a more rigorous assessment regime. The predicted postoperative $\mathrm{FEV}_{1}$ has been shown to be a particularly useful parameter in this setting, ${ }^{22}$ and this could be combined with $\mathrm{VO}_{2}$ measurements in patients undergoing pneumonectomy.

In conclusion, the shuttle walk test is a useful and readily available exercise test that assesses potentially operable lung cancer patients with borderline lung function. In these patients, distance performance on the shuttle walk test relates significantly to $\mathrm{VO}_{2}$ peak during treadmill testing. However, it tends to underestimate exercise capacity at the lower range compared with $\mathrm{VO}_{2}$ peak. Our findings also suggest that patients who exceed $400 \mathrm{~m}$ on the shuttle walk test may not need $\mathrm{VO}_{2}$ assessment. In patients failing to achieve this distance we would recommend $\mathrm{VO}_{2}$ assessment. This is particularly important in those unable to walk $250 \mathrm{~m}$ as many of these subjects had a $\mathrm{VO}_{2}$ peak in excess of $15 \mathrm{ml} /$ $\mathrm{kg} / \mathrm{min}$ and thus would have an acceptable surgical risk.

\section{Authors' affiliations}

T Win, C M Laroche, Thoracic Oncology Unit, Papworth Hospital, Papworth, Cambridge, UK

A Jackson, Respiratory Laboratory, Papworth Hospital, Papworth, Cambridge, UK

A M Groves, Radiology and Nuclear Medicine Department,

Addenbrooke's Hospital, Cambridge, UK

L D Sharples, S C Charman, MRC Biostatistics Unit, Robinson Way, Cambridge, UK

This work received funding from the Research and Development NHS Executive.

Competing interests: none.

\section{REFERENCES}

1 Semlik M, Schmid C, Trosch F, et al. Lung cancer surgery-preoperative risk assessment and patient selection. Lung Cancer 2001;33(Suppl 1):S9-15.

2 Weisman IM. Cardiopulmonary exercise testing in the preoperative assessment for lung resection surgery. Semin Thorac Cardiovasc Surg $2001 ; 13: 116-25$.

3 Schulz C, Emslander HP, Riedel M. Risk assessment of patients before lung surgery. Chirurgie 1999;70:664-73.

4 Bolliger CT, Wyser C, Roser $\mathrm{H}$, et al. Lung scintigraphy and ergospirometry in prediction of postoperative course in lung resection candidates with increased risk of postoperative complications. Pneumologie 1996;50:331-44.

5 Dales RE, Dionne G, Leech JA, et al. Preoperative prediction of pulmonary complications following thoracic surgery. Chest 1993;104:155-9.

6 Singh SJ, Morgan MDL, Scott S Walter D, et al. The development of the shuttlewalking test of disability in patients with chronic airway obstruction. Thorax 1992;47:1019-24.

7 Singh SJ, Morgan MDL, Hardman AE, et al. Comparison of oxygen uptake during a conventional treadmill test and the shuttle walking test in chronic airflow limitation. Eur Respir J 1994;7:2016-20.

8 British Thoracic Society. BTS guidelines on the selection of patients with lung cancer for surgery. Thorax 2001;56:89-108.

9 Beckles MA, Spiro SG, Colice GL, et al. American College of Chest Physicians. The physiologic evaluation of patients with lung cancer being considered for resectional surgery. Chest 2003;123:105-14S.

10 Solway S, Brooks D, Lacasse Y, et al. A quantitative systematic overview of the measurement properties of functional walk tests used in the cardio respiratory domain. Chest 2001;119:256-70.

11 Association of Respiratory Technology and Physiology. Practical handbook of respiratory function testing. UK: Association of Respiratory Technology and Physiology, 1999.

12 Borg GA. Psychophysical bases of perceived exertion. Med Sci Sports Exerc 1982;14:377-81.

13 Northridge DB, Grant S, Ford I, et al. Novel exercise protocol suitable for use on treadmill or bicycle ergometer. Br Heart J 1990;64:313-6.

14 Day JR, Rossiter HB, Coats EM, et al. The maximally-attainable $\mathrm{VO}_{2}$ during exercise in humans: the peak vs. maximum issue. J Appl Physiol 2003;95:1901-7.

15 Bradley J, Howard J, Wallace E, et al. Reliability, repeatability and sensitivity of the modified shuttle test in adult cystic fibrosis. Chest 2000;117:1666-71.

16 Grant S, Corbett K, Amjad AM, et al. A comparison of methods of predicting maximum oxygen uptake. Br J Sports Med 1995;29:147-52.

17 Leger LA, Lambert J. A maximal multistage 20-m shuttle run test to predict $\mathrm{VO}_{2}$ max. Eur J Appl Physiol 1982;49:1-12.

18 American Thoracic Society. ATS statement: guidelines for the six-minute walk test. Am J Respir Crit Care Med 2002;166:111-7.

19 Brunelli A, Al Refai M, Monteverde M, et al. Stair climbing test predicts cardiopulmonary complications after lung resection. Chest 2002; 121:1106-10.

20 Booth S, Adams L. The shuttle walking test: a reproducible method for evaluating the impact of shortness of breath on functional capacity in patients with advanced cancer. Thorax $2001 ; 56: 146-50$.

21 Riley M, Northridge DB, Henderson E, et al. The use of an exponential protocol for bicycle and treadmill exercise testing in patients with chronic cardiac failure. Eur Heart J 1992;13:1363-7.

22 Ribas J, Diaz O, Barbera JA, et al. Invasive exercise testing in the evaluation of patients at high-risk for lung resection. Eur Respir J 1998;12:1429-35. 